\title{
The mediating effect of health anxiety in the relationship between functional somatic symptoms and illness behavior in Chinese inpatients with depression
}

Yue-Jiao Ma ${ }^{1}$, Dong-Fang Wang ${ }^{2}$, Ming Yuan ${ }^{1}$, Jiang Long ${ }^{1,3}$, Shu-Bao Chen ${ }^{1}$, Qiu-Xia Wu ${ }^{1}, X^{\prime}$-Yi Wang ${ }^{1}$ and Tie-Qiao Liu ${ }^{1 *}$

\begin{abstract}
Background: Functional somatic symptoms in depression disorder may cause inappropriate illness behavior hindering the treatment process. Health anxiety may play a role in this relationship, but few studies have examined it. The current study aimed to investigate the role of health anxiety in the relationship between functional somatic symptoms and illness behavior in patients with depression.

Methods: The present study recruited 323 hospitalized patients with depression to complete the Patient Health Questionnaire-15, Whiteley-Index-7, and Scale for the Assessment of Illness Behavior, then constructed a structural equation model to examine whether health anxiety mediated the relationship between functional somatic symptoms and illness behavior.

Results: The results showed significant correlations between any two of the three variables of interest. More importantly, health anxiety played a partially mediating role (42.86\%) in the relationship between functional somatic symptoms and illness behavior. Further analysis suggested that elderly patients reached higher health anxiety than younger patients when their functional somatic symptoms were mild.
\end{abstract}

Conclusions: These results highlight that health anxiety may mediate the influence of functional somatic symptoms on illness behavior. The implications of assessing and intervening in health anxiety in patients with depression were discussed.

Keywords: Depression, Functional somatic symptoms, Illness behavior, Health anxiety, Mediating effect

\section{Background}

Somatic symptoms (SSs) without physical cause are very common in mental illness, such as depression disorder [1-4]. The proportion of functional SSs (FSSs) can reach up to $80 \%$ in patients with depression [5]. Most patients with depression incorrectly attribute FSSs to physical conditions, reporting somatic rather than emotional symptoms in their primary care [6]. Consequently,

\footnotetext{
* Correspondence: liutieqiao123@csu.edu.cn

'Department of Psychiatry, The Second Xiangya Hospital, Central South University, The China National Clinical Research Center for Mental Health Disorders, Chinese National Technology Institute of Psychiatry, Key Laboratory of Psychiatry and Mental Health of Hunan Province, No. 139, Middle Renmin Road, Changsha, Hunan 410011, People's Republic of China Full list of author information is available at the end of the article
}

patients with depression frequently use inappropriate forms of illness behavior (IB)-namely, the actions taken to deal with the health issue-such as frequent doctor visits and asking for more body scanning [7-9], treatment nonadherence and more frequent polypharmacy [10], and higher rates of unemployment [1]. This misdirecting or unnecessary IB can probably result in misdiagnosis, treatment delays, rehabilitation barriers, and huge waste of health care services $[8,11,12]$. Therefore, it would be very helpful to clarify the mechanism of how FSSs affect IB.

Health anxiety (HA) may play an important role in the relationship between FSSs and IB. HA is defined as the extensive worry that people can have about their health 
[13] with intensity ranging from none to severe; hypochondriasis (in the Diagnostic and Statistical Manual of Mental Disorders, Fourth Edition [DSM-IV]) or illness anxiety disorder (in the DSM-V) is an extreme form [14]. FSSs are strongly positively correlated with HA with evidence that patients with severe FSSs often accompanied by higher HA [15-17] tend to worry about their bodily signs and other ambiguous health-related symptoms [18]. In addition, evidence shows that higher HA can cause more inappropriate IB $[16,19,20]$. Therefore, it is worth examining whether HA functions as an intermediary mechanism between FSSs and IB.

The role HA plays between FSSs and IB may be that of a mediator. Some researchers have proposed that IB in patients with FSSs may depend on the underlying psychological pathology, which is closely related to HA, rather than the FSSs themselves [9]. In addition, previous studies showed that both FSSs and HA can predict IB [1, 9, 16]. FSSs appear to share a common neurologic pathway with depression, which is based on neurotransmitter dysregulation of serotonin and norepinephrine in depression disorder [21]. HA affects functional physical symptoms, but it is not the main factor of FSSs. On the contrary, the degree of FSSs will aggravate the level of HA [15], and HA is more contingent and can be changed, for example, by cognitive-behavioral therapy [18]. Thus, it is reasonable to assume that the affecting direction between FSSs and HA should be from the former to the latter rather than the reverse. Even though the relationships among FSSs, HA, and IB have received attention, few empirical studies have directly tied these variables together to examine the possible intermediary processes.

Accordingly, we propose the main hypothesis of the present study-namely, that the influence of FSSs on IB in patients with depression is probably mediated by their HA. Specifically, severe FSSs may produce higher HA, further causing more abnormal IB. To verify the hypothesis, we recruited a sample of Chinese inpatients with depression, whose non-Western cultural context may be associated with higher rates of somatic presentation than in Western or developed countries [22], and built a structural equation model based on structured measurements. We were also interested in whether patient age would have moderating effects in the mediation model of the above hypothesis as previous studies have shown that age-related differences exist in HA [23], medical utilization [24], and help-seeking behavior [25].

\section{Methods}

\section{Participants and procedure}

In this cross-sectional study, 323 inpatients with depression were recruited using convenient sampling from two large hospitals in China from April 2017 to March 2018:
The Second Xiang-Ya Hospital, Central South University, and the Second Affiliated Hospital of Guizhou Medical University. All patients enrolled needed to meet the recruitment criteria: 1) diagnosis of major depressive disorder according to the DSM-V by two psychiatrists with more than five years' clinical experience and 2) ability to understand the contents of all measures. Exclusion criteria were as follows: 1) comorbidity with bipolar disorder, schizophrenia, alcohol/substance use disorders, and other psychotic disorders and 2) major medical abnormalities, including central nervous system diseases or acute, unstable, or life-threatening medical illnesses (e.g., cancer, infections). All participants provided signed, informed consent to participate in this study. Ethical approval was obtained from the Mental Health Institute of Central South University Second Xiang-Ya Hospital.

Following the completion of informed consent forms, patients filled in a series of questionnaires including the Patient Health Questionnaire (PHQ-15), the scale for the Assessment of Illness Behavior (SAIB), and the Whiteley Index-7 (WI-7). Basic information such as gender, age, education, and illness duration was extracted from the patients' medical records.

\section{Measures \\ Functional somatic symptoms}

FSSs were assessed with the Chinese version of the PHQ-15, which was translated and revised by Lee et al. [26] from the original version [27]. The questionnaire involves 15 common SSs that account for more than $90 \%$ of the symptoms seen in primary care. The patients were asked to rate the severity of their symptoms during the past 4 weeks on a 3-point Likert scale: 0 ("not bothered at all"), 1 ("bothered a little"), or 2 ("bothered a lot"). Thus, the total symptom severity score varied from 0 to 30 with higher scores indicating severer SSs. The Chinese version of the PHQ-15 exhibited satisfactory internal consistency (Cronbach's alpha $=0.79$ ) [26]. The Cronbach's alpha in the present study was 0.86 .

\section{IIIness behavior}

IB was assessed with the Chinese version of the SAIB [28], which was translated and revised from the original version developed by Rief et al. [29]. The Chinese version of the SAIB is a 23-item self-report questionnaire composed of 5 subscales of diagnosis verification (e.g., concerning my diagnosis, I always ask for a second medical opinion), expression of symptoms (e.g., I often try to explain my current state of health to other people), medication/treatment (e.g., due to my complaints, I have already tried alternative medical treatments), illness consequences (e.g., I am not able to concentrate on my work when suffering from physical complaints), and body scanning (e.g., I pay a lot of attention to the 
different processes going on within my body). Each item is scored on a 4-point Likert scale that varies from 0 to 3 (0 = "I agree completely"; 1 = "I partially agree"; 2 = " I partially disagree"; 3 = "I disagree completely"). Lower scores represent severer dysfunctional IB. Internal consistency of the Chinese version of the SAIB was found to be 0.88 , and Cronbach's alphas across the 5 subscales were between 0.61 to 0.82 . Cronbach's alpha values in the present study were between 0.53 to 0.83 , and 0.88 for the total score on the SAIB.

\section{Health anxiety}

The Chinese version of the WI-7 [30], which was originally developed by Fink et al. [31], was used to assess HA. This questionnaire consists of 7 items, and each item has a dichotomous choice of "no" or "yes" $(0=$ "yes "; 1 = "no"). The total score (range 0-7) was obtained additively; higher scores indicate higher HA. The Chinese version of the WI-7 exhibited satisfactory internal consistency (Cronbach's alpha =0.73) [30]. The Cronbach's alpha in the present study was 0.81 .

\section{Statistical analyses}

Descriptive statistics and Pearson's correlation analyses of three variables (FSS, HA, and IB) were conducted with SPSS 22.0 software. The mediating role of HA in the relationship between FSSs and IB was tested using structural equation modeling (SEM) in AMOS 21.0 with the maximum likelihood estimation method conducted for model estimation. The goodness of fit of the model was evaluated using the following indices: CMIN/DF (a value between 1 and 5 indicates acceptable fit between hypothetical model and sample data), RMSEA $(<0.1$ reflects reasonable model fit) [32], SRMR $(<0.08$ indicates acceptable fit) [33], and CFI (>0.9 indicates acceptable fit) [34]. A multivariate regression analysis of FSS, HA, IB, and age was performed with Hayes' SPSS-PROCESS program model 59 to examine the adjusted moderating effect model of age [35]. Bootstrapped method was used to estimate the significance of indirect effects by 5000 bootstraps and 95\% confidence interval. $P$-values $<0.05$ were considered statistically significant.

\section{Results}

\section{Sample characteristics}

A total of 323 inpatients with depression were collected in our study; 153 (47.7\%) were female. Age ranged from 14 to 68 years, and the mean \pm SD of the total sample was $33.84 \pm 12.35$ years $(34.16 \pm 12.91$ in male and $33.48 \pm 11.72$ in female participants). The duration of education was $12.60 \pm 3.30$ years. The mean duration of illness (according to electronic medical records) was $21.8 \pm 47.17$ months.

\section{Correlational analyses}

Table 1 shows the results of the correlational analyses. The PHQ-15 score was negatively correlated with the SAIB score $(r=-0.50)$ and its five subscales $(r=-0.32 \sim$ -0.47), which means that the severer the SSs, the severer the dysfunctional IB. The PHQ-15 score was positively correlated with the WI-7 score $(r=0.53)$. In addition, WI-7 also negatively correlated with SAIB ( $r=$ $-0.53)$ and its five subscales $(r=-0.21 \sim-0.55)$, which means that people who have higher HA will have severer dysfunctional IB. The above results support the hypothesis of relationship among the three, and we conducted an intermediary model to further validate the hypothesis.

\section{Structural equation modeling}

SEM was used to explore the mediating role of HA in the relationship between FSSs and IB. Most of the fit indicators $(\mathrm{CMIN} / \mathrm{DF}=4.89, \mathrm{IFI}=0.93, \mathrm{NFI}=0.91, \mathrm{CFI}=0.93$, SRMR $=0.05)$ were acceptable, except $\mathrm{RMSEA}=0.11$. Therefore, we modified the model according to modification indices, revealing correlation between error3 and error4, which were latent variables of IB. After updating the model, the mediation model (see Fig. 1) showed an acceptable fit $(\mathrm{CMIN} / \mathrm{DF}=3.79, \mathrm{IFI}=0.95, \mathrm{NFI}=0.94, \mathrm{CFI}=$ 0.95 , SRMR $=0.04$, RMSEA $=0.09$ ), and all path coefficients were significant at the 0.001 level. The significance of the mediating effects of HA was tested by the bootstrap estimation procedure in AMOS (a bootstrap sample of 5000 was specified). As displayed in Table 2, SSs exerted significant indirect effects on IB via HA. HA also had a partial mediating effect in the relationship between SSs and IB; the indirect effects accounted for $42.86 \%$ (Indirect effect/Total effect) of the total effect.

\section{Moderate mediation model}

The PHQ-15 score was set as an independent variable and SAIB total score as a dependent variable, the WI7 score had a mediating effect, and age was a moderating variable. Multiple regression analysis was performed to test which path in the mediation model was adjusted. The result showed that the effect of age on the relationship between FSSs and HA was statistically significant. However, there was no statistically significant effect of the moderating in the relationship between $\mathrm{HA}$ and IB, and there was also no statistically significant moderating effect on the relationship between FSSs and IB (Table 3).

To further test the moderating effects of age between SSs and HA, subjects who aged above M + SD (33.84+ 12.35) were set as the high age group and below $M-S D$ (33.84-12.35) as the low age group. The results showed that the moderating effect was established in the low age group $($ effect $=0.48 ; 95 \%$ CI $[0.39 \sim 0.57])$, and the 
Table 1 Means, standard deviations, and correlations for all variables $(n=323)$

\begin{tabular}{|c|c|c|c|c|c|c|c|c|c|c|}
\hline & mean & SD & 1 & 2 & 3 & 4 & 5 & 6 & 7 & 8 \\
\hline 1.SAIB(Total) & 51.59 & 12.08 & 1 & & & & & & & \\
\hline 2.DV & 8.34 & 2.99 & $0.73^{* *}$ & 1 & & & & & & \\
\hline 3.ES & 12,16 & 3.40 & $0.69^{* *}$ & $0.33^{* *}$ & 1 & & & & & \\
\hline 4.MT & 13.00 & 3.95 & $0.77^{* *}$ & $0.44^{* *}$ & $0.44^{* *}$ & 1 & & & & \\
\hline $5.1 \mathrm{C}$ & 8.00 & 2.45 & $0.62^{* *}$ & $0.40^{* *}$ & $0.33^{* *}$ & $0.24^{* *}$ & 1 & & & \\
\hline 6.5 & 10.08 & 3.70 & $0.81^{* *}$ & $0.54^{* *}$ & $0.37^{* *}$ & $0.52^{* *}$ & $0.48^{* *}$ & 1 & & \\
\hline 7.PHQ-15 & 12.79 & 6.06 & $-0.50^{* *}$ & $-0.24^{* *}$ & $-0.32^{* *}$ & $-0.41^{* *}$ & $-0.36^{* *}$ & $-0.47^{* *}$ & 1 & \\
\hline 8.WI-7 & 5.14 & 2.07 & $-0.53^{* *}$ & $-0.39^{* *}$ & $-0.21^{* *}$ & $-0.37^{* *}$ & $-0.41^{* *}$ & $-0.55^{* *}$ & $0.53^{* *}$ & \\
\hline 9.age & 33.84 & 12.35 & $-0.17^{* *}$ & -0.03 & -0.11 & $-0.12^{* *}$ & -0.09 & $-0.17^{* *}$ & $0.17^{* *}$ & $020^{* *}$ \\
\hline
\end{tabular}

Note: M-means; SD—standard deviation

WI-7: Whiteley Index-7 (assessing health anxiety); SAIB: the Scale for the Assessment of Illness Behavior (assessing illness behavior); PHQ-15: the Patient Health Questionnaire (assessing somatic symptoms); DV: Diagnosis evaluation; ES: expression symptoms; MT: medication and treatment; IC: IIIness consequences;

S: Scanning;

**Significant at 0.01 level

moderating effect also existed in the high age group (effect $=0.29 ; 95 \%$ CI $[0.20 \sim 0.38]$ ). Furthermore, there was a significant difference between two age groups $(\mathrm{t}=-2.7585 ; p=0.0061)$. Those indicated a moderate-mediation model. (Fig. 2).

\section{Discussion}

The present study examined the role of HA in the relationship between FSSs and IB using SEM. The main hypothesis was partly supported by the results; HA was shown to partially mediate the association between FSSs and IB in a sample of the inpatients with clinical depression. More specifically, severer FSSs increased more abnormal IB by bringing about higher HA. We also found that age significantly moderated the impact of FSSs on HA with older patients experiencing higher HA for mild FSSs.

\section{Partial mediation of health anxiety}

Correlational analyses found an intensifying relationship between any two of FSS, HA, and IB. These findings are consistent with most previous literature suggesting that both elevated HA and severer SSs are significantly associated with more abnormal IB [9, 36-39] and with prior studies suggesting that severer SSs are linked to elevated HA $[15,17]$.

More importantly, according to the results of SEM, HA accounted for $42.86 \%$ of the total effect of FSSs on IB. In other words, increasing $\mathrm{HA}$ is an indirect approach of FSSs to elevate IB. To the best of our

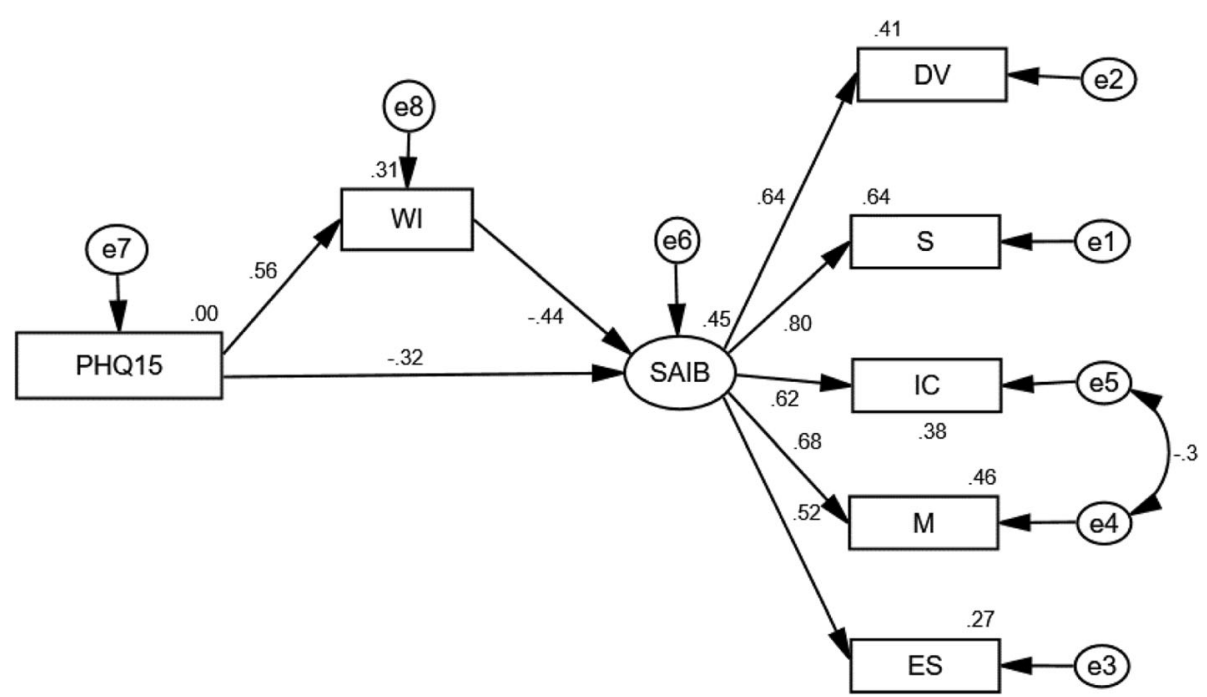

Fig. 1 The complete mediation model $(N=323)$. Path coefficients were standardized. Note: WI-7: Whiteley Index-7 (assessing health anxiety); SAIB: the Scale for the Assessment of Illness Behavior (assessing illness behavior); PHQ-15: the Patient Health Questionnaire (assessing somatic symptoms);DV: Diagnosis evaluation; ES: expression symptoms; MT: medication and treatment; IC: IIIness consequences; S: Scanning 
Table 2 Total effect, direct effect, indirect effect

\begin{tabular}{|c|c|c|c|c|}
\hline & Path & $\beta$ (standardization) & Bootstrop 95\% Cl & $P$ \\
\hline \multirow[t]{3}{*}{ PHQ-15 $\rightarrow$ SAIB } & Total effect (c) & -0.56 & $-0.63 \sim-0.48$ & $<0.001$ \\
\hline & Direct effect (c') & -0.32 & $-0.43 \sim-0.21$ & $<0.001$ \\
\hline & Indirect effect $(a \times b)$ & -0.24 & $-0.31 \sim-0.18$ & $<0.001$ \\
\hline
\end{tabular}

Note: SAIB: the Scale for the Assessment of Illness Behavior (assessing illness behavior); PHQ-15: the Patient Health Questionnaire (assessing somatic symptoms)

knowledge, this is the first study verifying the mediating role of HA between FSSs and IB and clarifying the portion of this mediating effect in the total effect of FSSs. Given that the remaining total effect may consist of a direct effect of FSSs and indirect effects of other variables beyond the present study's interest, this finding emphasizes that $\mathrm{HA}$ is an important intermediate mechanism when we consider how FSSs influence IB.

The sample of the present study was inpatients with depression, most of them with FSSs, which should be noted to understand the results. It is well known that depression is often accompanied by FSSs [1, 3]. Depression and anxiety often accompany each other, and severe HA was suggested to be reclassified as an anxiety disorder in the DSM-V [40]. Hence, we speculate that patients with depression may have higher anxiety qualities than other people. These people are more sensitive and excessively attend to their own specific signs and symptoms, subsequently worrying about their health and attributing these FSSs to organic causes rather than functional ones, resulting in elevated HA. Typically, patients with high HA tend to hold the catastrophic interpretation with FSSs (this headache means a brain tumor) [41]. To seek reassurance about the fear of such symptoms and to alleviate this kind of HA, patients often enact inappropriate IB [36, 42]. Therefore, it seems reasonable that $\mathrm{HA}$ would be a potent mediating factor of this association between FSSs and IB.

\section{Age effect}

Another interesting finding was that the age of patients with depression played a moderating role in the relationship between FSSs and HA. Although there were significant positive relationships between FSSs and HA in both of younger and older groups, a more thorough analysis revealed the age effect. Specifically, compared with the younger group, the older patients had higher HA when FSSs were mild. Given that both groups reached the same levels of HA when FSSs were severe, this pattern of age effect suggests that older patients with depression may overreact to mild FSS.

It is necessary to contrast the age effect found in the present study with the findings of previous studies. Previous research showed that older adults (over age 64) report relatively low levels of worrying about health issues than do college students [43]. The current research indicated that level of HA was higher in the high-aged group $(33.84+12.35)$ than in the low-aged group (33.84$12.35)$, but this growth trend gradually slowed with increasing age. The explanation for the inconsistent result may be that our study focused on a depressed population who may have high anxiety characteristics while the prior research focused on the general population. Another hypothesis from other researchers is that HA may peak at a certain age and decrease thereafter [44, 45]. Our study has an age range from 14 to 68 years; therefore, it is necessary to investigate in a larger age range allowing for comparison across the entire lifespan.

Table 3 Multiple regression analysis results with moderate mediation

\begin{tabular}{|c|c|c|c|c|c|c|c|c|}
\hline & \multicolumn{4}{|c|}{ Dependent variable:Wl } & \multicolumn{4}{|c|}{ Dependent variable:SAIB } \\
\hline & coef & SE & $\mathrm{t}$ & $\mathrm{p}$ & coef & SE & $\mathrm{t}$ & $p$ \\
\hline PHQ15 & 0.386 & 0.033 & 11.639 & 0.001 & & & & \\
\hline AGE & 0.002 & 0.001 & 2.432 & 0.016 & & & & \\
\hline PHQ15*AGE & -0.008 & 0.003 & -0.2836 & 0.005 & & & & \\
\hline WI & & & & & -0.641 & 0.100 & -.6 .411 & 0.001 \\
\hline AGE & & & & & -0.002 & 0.002 & -1.057 & 0.291 \\
\hline$W I * A G E$ & & & & & -0.001 & 0.008 & -0.078 & 0.938 \\
\hline PHQ15 & & & & & -0.370 & 0.071 & -5.252 & 0.001 \\
\hline AGE & & & & & -0.002 & 0.002 & -1.057 & 0.291 \\
\hline PHQ15*AGE & & & & & -0.003 & 0.006 & -0.474 & 0.636 \\
\hline
\end{tabular}

Note: WI-7: Whiteley Index-7 (assessing health anxiety); SAIB: the Scale for the Assessment of Illness Behavior (assessing illness behavior); PHQ-15: the Patient Health Questionnaire (assessing somatic symptoms) 


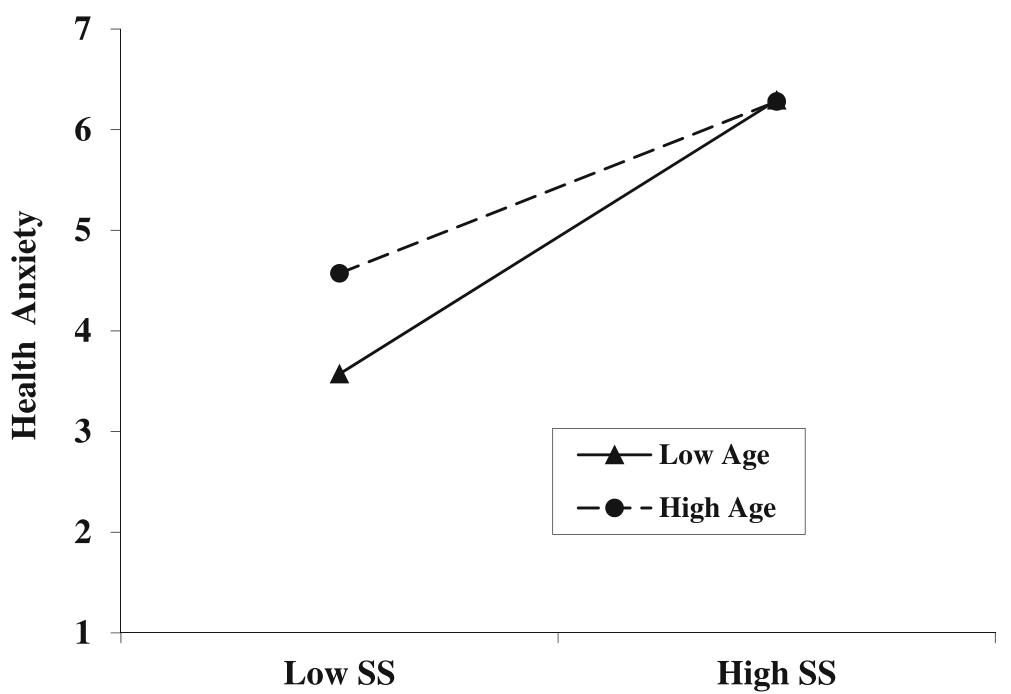

Fig. 2 The moderating effect of age on the association between FSS and HA. Note: The ordinate is the total score of health anxiety, and the abscissa: low SS refers to somatic symptoms (M-SD), High SS refers to somatic symptoms (M + SD); Low Age: age (M-SD), High Age: age (M + SD)

\section{Implications for treatment}

The results of the present study have multifaceted value for clinical practice with patients with depression disorder. First, the partially mediating role of HA between FSSs and IB highlights the need to pay more attention to HA (especially in high-aged patients with depression with SSs, even if mild) when treating depression disorder rather than dealing only with the depressive symptoms. Second, the results suggest that HA can be a promising intervention target for reducing depressive patients' inappropriate IB. Therefore, we recommend a regular assessment of FSSs and HA in patients with depression. For those with mild HA, the doctor should explain the nature of the symptoms to the patient, thereby avoiding more unnecessary HA and IB. For those with severe HA, timely and effective intervention is necessary. In addition, it is recommended to actively evaluate and intervene in HA in elderly patients with depression with FSS (even if mild).

Currently, the effectiveness of antidepressants in treating FSSs is far from ideal; there are often residual physical symptoms $[46,47]$. Studies have shown that the treatment response of HA was much better than FSSs [48]. Thus, the HA intervention becomes more important and efficient in reducing the IB of patients with depression. High levels of health anxiety might be a multidimensional trait, where triggering factor (such as physiological processes), cognitive and behavioral strengthen each other. Although avoiding triggers and using safe behaviors (such as more examinations of symptoms) may lead to a reduction in health anxiety in the short term but higher levels of health anxiety in the long run [49]. Fortunately, psychoeducation, exposure and response prevention, antidepressants, and cognitive restructuring techniques might be helpful for patients with severe health anxiety. Specifically, cognitive-behavioral therapy has been reported to be a highly effective treatment for hypochondriasis/HA $[18,50,51]$, even better than drug treatment [52]. The strengths of the cognitive behavioral model of severe health anxiety lies in its account of maintaining factors. The maintenance factor of health anxiety lies in the faulty cognitive belief of bodily sensations and external events. Consequently, the hallmark of cognitive therapy is that it entails components aiming to change these faulty beliefs. It can alleviate $\mathrm{HA}$ by helping patients recognize and modify false beliefs about the symptoms [41] . A recent study found that cognitive-behavioral therapy can reduce perceived risk of disease, attention to bodily symptoms, and intolerance of uncertainty significantly to improve HA [53].

\section{Limitations}

A number of limitations of the present study should be considered. First, given the cross-sectional design, the current study could not infer the causal nature of the associations among FSS, HA, and IB. Thus, future study should examine the associations of these variables using a longitudinal design or an experimental manipulation method that allows causal relationship. Second, the present study examined the mediating role of HA in the relationship between FSSs and IB in a clinical depressed sample, whether this conclusion can be extended to other mental disorders requires further confirmation. Therefore, future research should utilize a longitudinal 
design to examine this association in other samples to determine the clinical relevance of the findings. Third, we did not consider other factors that previous studies have reported that may affect IB, such as personality traits [54] and depression [7] . It is worth mentioning that we found no association between SAIB and the depression scale of Patients Health Questionnaire-9 in the present study, which is consistent with the finding of Rief et al. [29]. While this result is in contrast to results of Wilson Barnett and Trimble who using Illness Behavior Questionnaire (IBQ) as assessment tool [55]. The possible reason for this inconsistence may be that IBQ focus on evaluation of emotional aspects and hypochondriacal concerns rather than aspects of illness behavior (SAIB). However, because the subjects recruited are hospitalized patients with severe symptoms of depression in our study, depression may still have certain effects on variables in the mediation model. It would be helpful to validate the mediation model in the general population. Despite these limiting factors, the strength of this study is the emphasis on the mediating effect of health anxiety in clinical major depressed samples. In future, the result can be further validated in a population-based study and non-hospitalized sample in order to control these effects.

\section{Conclusion}

In conclusion, the current study reports the partial mediating role of $\mathrm{HA}$ in the relationship between FSSs and IB in a Chinese sample of inpatients with depression, which was moderated by age. The results of the present study emphasize the importance of HA in patients with depression with SSs, and it is necessary to evaluate and intervene in HA appropriately, especially in older patients.

\section{Abbreviations}

FSS: functional somatic symptoms; HA: Health anxiety; IB: Illness behavior IBQ: IIIness Behavior Questionnaire; PHQ-15: Patient Health Questionnaire; SAIB: The scale for the Assessment of Illness Behavior; SS: Somatic symptoms; WI-7: The Whiteley Index-7

\section{Acknowledgements}

We sincerely thank all those have contributed to this article. Thanks to Hilary from Editage for the grammar editing of this article.

\section{Authors' contributions}

TQL were responsible for study design, manuscript preparation and revision. YJM and DFW were responsible for data collection, statistical analysis, manuscript preparation and writing the protocol and the paper. MY had a guiding role for the writing of this article. JL, SBC, QXW and XYW participated in the design of the study and in the acquisition of data. All authors have contributed to and have approved the final manuscript.

\section{Authors' information}

Not applicable.

\section{Funding}

This work was supported by grants from the National Key R\&D Program of China (2017YFC1310400) and the National Natural Science Foundation of China (81371465 and 81671324). The funders had no role in study design, data collection and analysis, decision to publish, or preparation of the manuscript.

\section{Availability of data and materials}

The datasets used and/or analyzed during the current study are available from the corresponding author on reasonable requests.

\section{Ethics approval and consent to participate}

This study was approved by the Mental Health Institute of Central South University Xiang-Ya Second Hospital. All subjects signed an informed consent to participate in this study, which was carried out in accordance with the Helsinki Declaration.

Consent for publication

Not applicable.

\section{Competing interests}

The authors declare that they have no competing interests.

\section{Author details}

${ }^{1}$ Department of Psychiatry, The Second Xiangya Hospital, Central South University, The China National Clinical Research Center for Mental Health Disorders, Chinese National Technology Institute of Psychiatry, Key Laboratory of Psychiatry and Mental Health of Hunan Province, No. 139, Middle Renmin Road, Changsha, Hunan 410011, People's Republic of China. ${ }^{2}$ Psychosomatic health institute of the Third Xiangya Hospital, Central South University, Changsha 410013, Hunan, People's Republic of China. ${ }^{3}$ Laboratory for Experimental Psychopathology, Psychological, Science Research Institute, Université Catholique de Louvain, Louvain-la-Neuve, Belgium.

Received: 13 May 2019 Accepted: 19 August 2019

Published online: 27 August 2019

\section{References}

1. Kapfhammer HP. Somatic symptoms in depression. Dialogues Clin Neurosci. 2006;8(2):227-39.

2. Wang J, Guo WJ, Mo LL, Luo SX, Yu JY, Dong ZQ, Liu Y, Huang MJ, Wang Y, Chen L. Prevalence and strong association of high somatic symptom severity with depression and anxiety in a Chinese inpatient population. Asia-Pacific psychiatry : official journal of the Pacific Rim College of Psychiatrists. 2017;9(4). https://doi.org/10.1111/appy.12282.

3. Greco T, Eckert $G$, Kroenke $K$. The outcome of physical symptoms with treatment of depression. J Gen Intern Med. 2004;19(8):813-8.

4. Kroenke K. Patients presenting with somatic complaints: epidemiology, psychiatric comorbidity and management. Int J Methods Psychiatr Res. 2003:12(1):34-43.

5. Hamilton M. Frequency of symptoms in melancholia (depressive illness). $\mathrm{Br}$ J Psychiatry. 1989;154(2):201-6.

6. Tylee A, Gandhi P. The importance of somatic symptoms in depression in primary care. Prim Care Companion J Clin Psychiatry. 2005;7(4):167-76.

7. Rief W, Martin A, Klaiberg A, Brähler E. Specific effects of depression, panic, and somatic symptoms on illness behavior. Psychosom Med. 2005;67(4): 596-601.

8. Bao $Y$, Sturm R, Croghan TW. A national study of the effect of chronic pain on the use of health care by depressed persons. Psychiatr Serv. 2003;54(5):693.

9. Guo Y, Kuroki T, Koizumi S. Abnormal illness behavior of patients with functional somatic symptoms: relation to psychiatric disorders. Gen Hosp Psychiatry. 2001;23(4):223-9.

10. Keeley R, Smith M, Miller J. Somatoform symptoms and treatment nonadherence in depressed family medicine outpatients. Arch Fam Med. 2000:9(1):46-54

11. Barsky AJ, Orav EJ, Bates DW. Somatization increases medical utilization and costs independent of psychiatric and medical comorbidity. Arch Gen Psychiatry. 2005;62(8):903-10.

12. Luber MP, Meyers BS, Williams-Russo PG, Hollenberg JP, Didomenico TN, Charlson ME, Alexopoulos GS. Depression and service utilization in elderly primary care patients. American Journal of Geriatric Psychiatry Official Journal of the American Association for Geriatric Psychiatry. 2001;9(2):169. 
13. Watt MC. Review of It's not all in your head: how worrying about your health could be making you sick--and what you can do about it. Can Psychol. 2010;47(3):235-7.

14. Starcevic V. Hypochondriasis and health anxiety: conceptual challenges. Br J Psychiatry. 2013;202(1):7.

15. Lee $\mathrm{S}, \mathrm{Creed} \mathrm{FH}, \mathrm{Ma} \mathrm{YL}$, Leung $\mathrm{CM}$. Somatic symptom burden and health anxiety in the population and their correlates. J Psychosom Res. 2015;78(1): 71-6.

16. Creed F. The relationship between somatic symptoms, health anxiety, and outcome in medical out-patients. Psychiatr Clin N Am. 2011;34(3):545-64.

17. Clarke DM, Piterman L, Byrne CJ, Austin DW. Somatic symptoms, hypochondriasis and psychological distress: a study of somatisation in Australian general practice. Med J Aust. 2008;189(10):560-4.

18. Olatunji BO, Kauffman BY, Meltzer S, Davis ML, Smits JA, Powers MB. Cognitive-behavioral therapy for hypochondriasis/health anxiety: a metaanalysis of treatment outcome and moderators. Behaviour Research \& Therapy. 2014;58(4):65-74.

19. Fink P, Ørnbøl E, Christensen KS. The Outcome of Health Anxiety in Primary Care. A Two-Year Follow-up Study on Health Care Costs and Self-Rated Health. Plos One. 2010;5(3):e9873.

20. Skidmore JR, Dyson SJ, Kupper AE, Calabrese D. Predicting illness behavior: health anxiety mediated by locus of control. Am J Health Behav. 2014;38(5): 699-707.

21. Trivedi $\mathrm{MH}$. The link between depression and physical symptoms. Prim Care Companion J Clin Psychiatry. 2004;6(Suppl 1):12-6.

22. Katon W, Kleinman A, Rosen G: Depression and somatization: a review, Part American Journal of Medicine 1982, 72(1):127.

23. Gerolimatos LA. Age-related differences in the experience of health anxiety and use of coping strategies. Dissertations \& Theses - Gradworks. 2014.

24. Fergus TA, Griggs JO, Cunningham SC, Kelley LP. Health anxiety and medical utilization: the moderating effect of age among patients in primary care. Journal of Anxiety Disorders. 2017:51.

25. Koloski NA, Talley NJ, Boyce PM. Epidemiology and health care seeking in the functional GI disorders: a population-based study. Am J Gastroenterol. 2002;97(9):2290-9.

26. Lee S, Ma YL, Tsang A. Psychometric properties of the Chinese 15-item patient health questionnaire in the general population of Hong Kong. J Psychosom Res. 2011;71(2):69-73.

27. Kroenke K, Spitzer RL, Williams JB. The PHQ-15: validity of a new measure for evaluating the severity of somatic symptoms. Psychosom Med. 2002; 64(2):258.

28. Dongfang W, Rui L, Huiqin L, Yanqi L, Yunlong D. Reliability and validity of the Chinese-version of the assessment of illness behavior in medical college students. Chin J Behav Med \& Brain Sci. 2018;17(5):466-9

29. Rief W, Ihle D, Pilger F. A new approach to assess illness behaviour. J Psychosom Res. 2003;54(5):405-14.

30. Lee $\mathrm{S}, \mathrm{Ng} \mathrm{KL}$, Ma YL, Tsang A, Kwok KP. A general population study of the Chinese Whiteley-7 index in Hong Kong. J Psychosom Res. 2011 71(6):387-91.

31. Fink $P$, Ewald $H$, Jensen J, Sørensen L, Engberg M, Holm M, Munk-Jørgensen $P$ : Screening for somatization and hypochondriasis in primary care and neurological in-patients: a seven-item scale for hypochondriasis and somatization 1999, 46(3):261-273.

32. Steiger JH. Structural model evaluation and modification: an interva estimation approach. Multivariate Behav Res. 1990;25(2):173-80.

33. Hu LT, Bentler PM. Fit indices in covariance structure modeling: sensitivity to Underparametrized model Misspesification. Psychol Methods. 1998:3(4):424-53.

34. Bentler PM. Comparative fit indexes in structural models. Psychol Bull. 1990; 107(2):238.

35. Hayes A . Introduction to mediation, moderation, and conditional process analysis[J]. J Educ Meas, 2013, 51(3):335-337.

36. Weiss FD, Rief W, Martin A, Rauh E, Kleinstäuber M. The heterogeneity of illness behaviors in patients with medically unexplained physical symptoms. International Journal of Behavioral Medicine. 2016;23(3):319-26.

37. Tomenson B, Mcbeth J, Chew-Graham CA, Macfarlane G, Davies I, Jackson J, Littlewood A, Creed FH. Somatization and health anxiety as predictors of health care use. Psychosom Med. 2012;74(6):656-64.

38. Kapur N, Hunt I, Lunt M, Mcbeth J, Creed F, Macfarlane G. Psychosocial and illness related predictors of consultation rates in primary care--a cohort study. Psychol Med. 2004;34(4):719-28.
39. Jyväsjärvi S, Joukamaa M, Väisänen E, Larivaara P, Kivelä S, Keinänenkiukaanniemi S. Somatizing frequent attenders in primary health care. J Psychosom Res. 2001;50(4):185-92.

40. APA: Diagnostic and Statistical Manual of Mental Disorders, Fifth Edition (DSM-5 ${ }^{\oplus}$ ): Naklada Slap; 2014.

41. Taylor S. Understanding and treating health anxiety: a cognitive-behavioral approach[J]. Cognitive \& Behavioral Practice. 2005;11(1):112-23.

42. Rief W, Hiller W, Margraf J. Cognitive aspects of hypochondriasis and the somatization syndrome. J Abnorm Psychol. 1998;107(4):587.

43. Hunt $S$, Wisocki P, Yanko J. Worry and use of coping strategies among older and younger adults. J Anxiety Disord. 2003;17(5):547-60.

44. Knauer SR, Freburger JK, Carey TS. Chronic low back pain among older adults: a population-based perspective. J Aging Health. 2010;22(8):1213-34.

45. Cornally N, Mccarthy G. Help-seeking behaviour for the treatment of chronic pain. British Journal of Community Nursing. 2011;16(2):90.

46. Kennedy N, Paykel ES. Residual symptoms at remission from depression: impact on long-term outcome. J Affect Disord. 2004:80(2-3):135-44.

47. Fleck MPDA, Simon G, Herrman H, Bushnell D, Martin M, Patrick D. Major depression and its correlates in primary care settings in six countries. 9month follow-up study. Br J Psychiatry. 2005;186(1):41-7.

48. Barsky AJ, Ahern DK. Cognitive behavior therapy for hypochondriasis: a randomized controlled trial. Jama. 2004:291(291):1464-70.

49. Hedman E, Axelsson E. Severe health anxiety in the somatic symptom and related disorders[M]// treatments for psychological problems and syndromes. Ltd: John Wiley \& Sons; 2017.

50. Thomson AB, Page LA. Psychotherapies for hypochondriasis. Cochrane Database Syst Rev. 2007:4(4):CD006520.

51. Coons MJ, Asmundson GJG, Taylor S. Current Directions in the Treatment of Hypochondriasis. J Cogn Psychother. 2005;19(3):285-304. https://doi.org/1 0.1891/jcop.2005.19.3.285.

52. Walker J, Vincent N, Furer P, Cox B, Kjernisted K. Treatment preference in hypochondriasis. Journal of Behavior Therapy \& Experimental Psychiatry. 1999;30(4):251-8.

53. Hedman E, Lindefors $N$, Andersson G, Andersson E, Lekander M, Rück C, Ljótsson B. Predictors of outcome in internet-based cognitive behavior therapy for severe health anxiety. Behaviour Research \& Therapy. 2013; 51(10):711-7.

54. Michal M, Wiltink J, Grande G, Beutel ME, Brähler E. Type D personality is independently associated with major psychosocial stressors and increased health care utilization in the general population. J Affect Disord. 2011; 134(1-3):396-403

55. Wilson-Barnett J, Trimble MR. An investigation of hysteria using the illness behaviour questionnaire. British Journal of Psychiatry the Journal of Mental Science. 1985:146(6):601.

\section{Publisher's Note}

Springer Nature remains neutral with regard to jurisdictional claims in published maps and institutional affiliations.

Ready to submit your research? Choose BMC and benefit from:

- fast, convenient online submission

- thorough peer review by experienced researchers in your field

- rapid publication on acceptance

- support for research data, including large and complex data types

- gold Open Access which fosters wider collaboration and increased citations

- maximum visibility for your research: over $100 \mathrm{M}$ website views per year

At BMC, research is always in progress.

Learn more biomedcentral.com/submissions 